MODELO DE GESTIÓN ESTRATÉGICA EN UNIDADES DE EMPRENDIMIENTO EMPRESARIAL DE LA UNIVERSIDAD DE LA GUAJIRA ${ }^{1}$

\title{
MODEL OF STRATEGIC MANAGEMENT IN UNITS OF ENTREPRENEURSHIP AT THE UNIVERSIDAD DE LA GUAJIRA
}

\author{
Remedios Pitre Redondo ${ }^{2}$, Arnulfo Díaz Pertuz ${ }^{3}$ y Jaidith Eneth Vidal ${ }^{4}$ \\ Universidad de La Guajira (Colombia)
}

RECIBIDO: 16 de septiembre de 2014

ACEPTADO: 2 de diciembre de 2014

\section{RESUMEN}

El presente artículo ha sido derivado de un proyecto de investigación, cuyo objetivo general consistió en la construcción de un modelo de gestión estratégica aplicado al fortalecimiento de las estructuras organizacionales de las unidades de emprendimiento empresarial (MGEC) en el departamento de La Guajira. El abordaje metodológico fue de corte empírico-analítico de tipo, con un diseño transeccional no experimental. Para tal efecto, se diseñó una muestra probabilística de 367 participantes del nivel de educación superior estimada con $95 \%$ de confianza y $5 \%$ de error, a quienes se les administró una escala Likert sobre emprendimiento y empresarismo, la cual obtuvo un nivel de confiabilidad alpha de Cronbach ( $\alpha: .986)$. Los principales hallazgos permitieron identificar que a partir de la implementación del (MGEC) se evidenciaron cambios en las prácticas y factores asociados con la efectividad y operatividad de la formación para el emprendimiento y el empresarismo. Estos resultados constituyeron un punto de partida en la formulación de acciones estratégicas orientadas a la articulación de los ejercicios educativos con las políticas de desarrollo empresarial; replanteando su estructura y mejorando su proceso misional, y el reconocimiento de la importancia que tienen las competencias empresariales y personales en los procesos de liderazgo y creación de negocios de alta pertinencia y relevancia frente a las políticas de desarrollo y crecimiento económico del departamento de La Guajira.

Palabras clave: modelo, gestión estratégica, emprendimiento empresarial, educación superior, diseño organizacional, departamento de La Guajira.

\begin{abstract}
This article has been derived from a research project, whose overall goal consisted in the construction of a model of strategic management applied to the strengthening of the organizational structures of units of entrepreneurship (MGEC) in the department of La Guajira. The methodological approach of court was empirical-analytical type, with a transactional non-experimental design. For this purpose, we will design a probability sample of 367 participants of the level of higher education estimated with $95 \%$ confidence and 5\% error, who were administered a Likert scale on entrepreneurship and entrepreneurship, which obtained a level of reliability by Cronbach's Alpha ( $\alpha: .986)$. The major findings, allowed us to identify that depart from the implementation of the (MGEC) changes were evidenced in the practices and factors associated with the effectiveness and operation of the training for entrepreneurship and entrepreneurship. These results constitute a starting point for the formulation of strategic actions aimed at the articulation of learning exercises with the enterprise development policies; rethinking its structure and improve its missional process, and the recognition of the importance of business skills and personal in the processes of leadership and building a business of high relevance and relevance to the policies of economic growth and development of the department of La Guajira.
\end{abstract}

Keywords: Model, Strategic Management, Business Entrepreneurship, Higher Education, Organizational Design, Department of La Guajira.

Este Artículo se puede referenciar

Pitre, R., Díaz, A. \& Vidal, J. (2015) Modelo de gestión estratégica en unidades de emprendimiento empresarial de la universidad de la Guajira, en Desarrollo Gerencial Revista de la Facultad de Ciencias Económicas, administrativas y contables de la Universidad Simón Bolívar 7(1), 140-159.

\footnotetext{
${ }^{1}$ Proyecto de Investigación: Construcción de un modelo de gestión estratégica aplicado al fortalecimiento de las estructuras organizacionales de las unidades de emprendimiento empresarial (MGEC) en el departamento de La Guajira

${ }^{2}$ Magíster en Desarrollo y Gestión de Empresas Sociales, docente Tiempo Completo Universidad de La Guajira, remediopitre@hotmail.com

${ }^{3}$ Magíster en Desarrollo y Gestión de Empresas Sociales, docente catedrático de la Universidad de La Guajira, extensión Maicao, diazpertuz.2186@gmail.com

${ }^{4}$ Magíster en Desarrollo y Gestión de Empresas Sociales, docente Tiempo Completo Universidad de La Guajira, jvidal@uniguajira.edu.co
}

Desarrollo Gerencial, 7 (1) pp. 140 - 159 Enero-Junio 2015. ISSN: 2145-5147 (On Line). Universidad Simón Bolívar. 


\section{1.- INTRODUCCIÓN}

Dentro del proceso de gestión y desarrollo de empresas sociales se planteó en su fase inicial, como núcleo problemático, el emprendimiento social. Desde esta perspectiva, se sientan las bases para liderar un proceso investigativo en el contexto sociolaboral de las universidades del departamento La Guajira. Se toma entonces, como realidad objeto de estudio a las universidades del departamento [Universidad de La Guajira y sus extensiones municipales, Universidad San Martín, Universidad de Pamplona, Universidad del Magdalena, Universidad Antonio Nariño, Universidad Nacional Abierta y a Distancia, Instituto Nacional de Formación Técnica Profesional (Infotep)], cuya realidad problemática nace al revisar la existencia y operatividad de las estructuras y/o unidades de emprendimiento y determinar su organización administrativa.

En este sentido, se evidencia que a partir de la promulgación de la Ley 1014/2006, el tema del emprendimiento ha sido el centro de interés tanto para la Universidad desde la misma formulación de su PEI como para el Departamento de La Guajira en sus planes de desarrollo; y aunque es un tema que encierra cierto grado de complejidad, siempre ha sido la base para el auge del desarrollo empresarial y el fomento de la cultura emprendedora.

Por tal motivo, el estímulo a la actividad emprendedora gira en torno a las políticas de desarrollo económico nacional y del departamento postuladas a lo largo de diferentes actos legislativos en la agenda de competitividad nacional y la agenda prospectiva del departamento (Congreso de la República de Colombia, 1990; 1996; 1999; 2000; 2002; 2004; 2006; Departamento Nacional de Planeación, 2007; 2010; Universidad de La Guajira, 2003; Gobernación de la Guajira, 2004; Sáenz \& Peña, 2006).

Desde lo anteriormente planteado surgen los siguientes interrogantes: ¿Cómo promover la construcción de un modelo de gestión estratégica para que la efectividad y operatividad de las estructuras y/o unidades de emprendimiento sea adecuado y genere cambios en las prácticas de formación para el emprendimiento y el empresarismo en La Guajira? ¿Cómo promover acciones estratégicas que lleven a articular las acciones educativas con las políticas de desarrollo empresarial del departamento? ¿Cómo motivar en el talento humano la formación para aprender a aprender del emprendimiento con el fin de gestar nuevas e innovadoras ideas de negocio?

Cada interrogante planteado conlleva establecer acciones en pro de transformar la gestión del emprendimiento, generar confianza y credibilidad, mejorar los procesos de formación del emprendedor, generar (gestionar) capital semilla y estructurar el laboratorio de incubación de empresas. Entonces, se trata de implementar un proceso de cambio que tiene como núcleo el conjunto de prácticas de los actores 


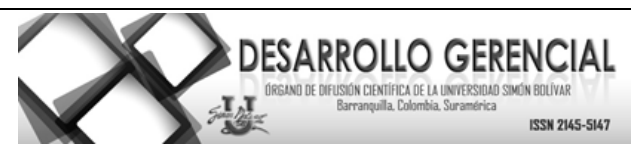

que permitan mejorar la eficacia y la eficiencia. Además, lograr la equidad, la pertinencia y la relevancia de la acción emprendedora.

Para afrontar este nuevo reto, se propone la creación de un centro de desarrollo empresarial que busca implementar ideas de negocio, la interacción de diversas actividades como asesoría, asistencia técnica, capacitación, gestión empresarial, capacitación técnica, consultoría, outsourcing, servicio de acceso al mercado, seguimiento y transferencia de tecnología. Estos ejes podrían ser considerados como elementos predictores del éxito en los procesos de emprendimiento empresarial

En este sentido el emprendimiento se ha acompañado del escenario contextual de las "spin-off universitarias", que pueden implementarse en diversos escenarios y aprovechar los resultados de grupos de investigación: una tesis de doctorado, un trabajo de grado o incluso un trabajo sobre alguna materia; también pueden llegar a ser desencadenantes de empresas, o soluciones de problemas planteados por los docentes en los laboratorios, o incluso llegar a ser innovaciones patentables.

Ello tiene en cuenta fundamentalmente un liderazgo compartido, trabajo colaborativo o una participación social responsable de los actores interinos de las universidades del departamento de La Guajira, articulado en los planteamientos sobre los procesos innovadores que deben ser abordados con el máximo nivel de alcance posible, según lo argumenta Mendoza (2006). Así, dentro del proceso de diseño del (MGEC) se desarrolló una primera etapa de investigación documental donde se revisaron diversas experiencias significativas de numerosas unidades de emprendimiento que se han creado en claustros de educación superior del país.

Tal es el caso de las universidades como del Norte, Eafit, Antioquia, Nacional, Simón Bolívar Amazonia, por lo que es fundamental dinamizar el accionar de la unidad y proponer el nuevo modelo de gestión estratégica para la operatividad de las estructuras y/o unidades de emprendimiento de las universidades del departamento de La Guajira (González, Ruiz, Orozco, Zuleta \& Morales, 2004; Institución Universitaria-ITM, 2010; Lanzas, Lanzas \& Castaño, 2006; Montañés \& Tovar, 2009; Universidad de Amazonia, 2011; Universidad de los Andes, Icesi, Universidad del Norte, 2006; Universidad Javeriana, 2010; Universidad de San Buenaventura, 2007 y Universidad de La Guajira, 2010).

De la misma manera, esta aproximación se argumenta con los aportes teóricos de Stiegelbauer (1990), Krishnaswami y Subramaniam (1999), Gámez (2009), Garzón (2008) y Torres (2008), quienes establecen que existen empresas de diferentes tipos y modelos administrativos innovadores desde los cuales se desarrollan tecnologías y procesos adaptados y ajustados a sus necesidades organizacionales. 
Esta es una de las formas en las que se materializa el modelo; pues en un contexto empresarial que asume el esquema de spin-off aquel puede surgir derivado de la evolución organizacional de una empresa, para mejorar su enfoque a partir de una relación funcional entre investigación universitaria y el sistema productivo que articule la transferencia de conocimientos generados y la presencia de las universidades en el proceso de innovación atendiendo especialmente la vinculación con el sistema productivo de su entorno (Universidad de Antioquia, 2012; Montoya, 2009; Navia \& Herrera, 2007; Rasmussen, 2006; Ruiz, 2009; Shane, 2004).

En este caso, dicha articulación podrá, llevarse a cabo a través de la creación de empresas de base tecnológica desde la actividad universitaria, donde cabe la participación del mismo personal docente e investigador del sector, y se pone en evidencia cuán importante es el desarrollo de modelos pedagógicos como estímulo a los procesos de emprendimiento en este ámbito, tal como lo proponen Andrade \& Bravo (2009).

Para hacer inferencia de las acciones administrativas que se dan dentro del contexto de las estructuras y/o unidades de emprendimiento, se han implementado algunas investigaciones interpretativas orientadas con estudio de casos (Yin, 1989), por lo cual, además, resulta imprescindible cualificar y cuantificar los comportamientos y actitudes de cada uno de los agentes que directa e indirectamente están vinculados a la estructura emprendedora universitaria, e identificar la población de estudiantes que a ella ingresan; también hay que determinar los tipos de proyectos que se postulan para incubación, y describir los procesos que se ponen en práctica para la búsqueda de capital semilla que garantice la respectiva financiación (Amit \& Muller, 1994; Araya, 2006).

\section{2.- MÉTODO}

El abordaje metodológico se asumió desde el paradigma empírico-analítico; en consecuencia, se empleó una aproximación cuantitativa (Kerlinger \& Lee, 2002) orientada en función del objetivo general y el abordaje del problema objeto de estudio relacionado con la necesidad de obtener información primaria que brinde elementos para sustentar la construcción de un modelo de gestión estratégica aplicado al fortalecimiento de las estructuras organizacionales de las unidades de emprendimiento empresarial (MGEC).

\section{Diseño}

El diseño metodológico empleado fue de tipo transeccional no experimental de corte descriptivo (Hernández, Fernández \& Baptista, 2003), sustentado en una etapa preliminar de investigación documental de fuentes primarias y secundarias asociadas con la formulación y diseño de modelos 


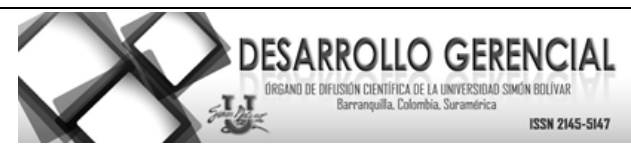

operativos en unidades de emprendimientos regionales en Colombia. De esta manera, en la segunda etapa se procedió posteriormente a la selección probabilística de la muestra de estudio identificada como segmento estratégico de las unidades de emprendimiento de la Universidad de La Guajira, a las que se les administró una encuesta tipo Likert.

\section{Participantes}

La muestra de estudio asumió un diseño de muestreo aleatorio simple y se conformó con 367 participantes miembros de la comunidad académica de la Universidad de La Guajira asumiendo un 95\% de confianza y $5 \%$ de error muestral (decanos, directores de programas, estudiantes emprendedores, docentes, emprendedores, directivos y personal administrativo de la misma institución educativa).

\section{Instrumentos}

Se diseñó una encuesta en escala Likert que recogió una serie de ítems relacionados con las expectativas y la aplicación del modelo, basándose en las teorías de referencia del modelo de spin-off, teoría de innovación, teoría de emprendimiento, teoría de cluster, articulada con el análisis de los factores asociados a los modelos existentes de las unidades de emprendimiento que actualmente se encuentran implementadas en diferentes universidades del país (Sampieri, Collado \& Baptista, 2003; Alburquerque \& Valdivieso, 2004; Amit \& Muller, 1994; Lanzas, Lanzas \& Lanzas, 2009; Ledesma, Molina, \& Valero, 2002; Rodeiro, Fernández, Otero \& Rodríguez, 2008).

Para efecto del procesamiento y análisis estadístico de la información, se utilizó el programa estadístico SPSS (Luna, Requena, Femia, Martin \& Miranda, 2007). A partir de la aplicación de la escala Likert del modelo, con la muestra de 367 participantes, se estimó su nivel de confiabilidad alpha de Cronbach, que resultó óptimo: un valor igual a ( $\alpha$ : .986).

\section{Procedimiento}

La investigación comprendió cuatro etapas: el análisis situacional, investigación documental de fuentes primarias y secundarias, investigación de campo con la técnica de encuesta tipo Likert y el procesamiento estadístico de la información.

\section{3.- RESULTADOS}

A continuación se presentan los principales hallazgos derivados del estudio llevado a cabo con la participación de los 367 funcionarios y estudiantes de la comunidad académica en la Universidad de La 


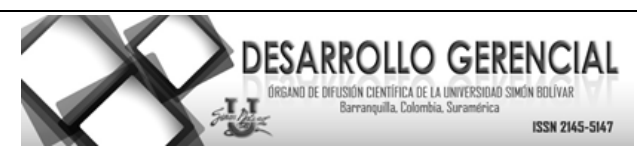

Guajira. En primera instancia, las principales tendencias asociadas con las expectativas frente al nuevo modelo de gestión operativa de la unidad de emprendimiento; posteriormente se identifican los efectos e incidencia del modelo, asociados con los procesos de incubación y desarrollo organizacional. Por último, se muestra un análisis de la correlación entre la percepción del modelo existente y las expectativas frente al nuevo modelo de gestión; entendido como un hallazgo de valor agregado (post hoc), el cual fue derivado del procesa miento complementario adelantado a partir del análisis de los datos, dado que el objeto central del estudio es de corte descriptivo.

Tabla1. Expectativas frente al nuevo modelo de gestión operativa de la Unidad de Emprendimiento

\begin{tabular}{lccc}
\hline \multicolumn{1}{c}{ Opinión } & Frecuencia & \% & \% Acumulado \\
\hline En desacuerdo & 81 & 22,1 & 22,1 \\
Ni de acuerdo, & 70 & 19,1 & 41,1 \\
ni en desacuerdo & & & \\
De acuerdo & 90 & 24,5 & 65,7 \\
Totalmente de acuerdo & 126 & 34,3 & 100,0 \\
\hline Total & $\mathbf{3 6 7}$ & $\mathbf{1 0 0 , 0}$ & \\
\hline
\end{tabular}

Fuente: autores (2012).

Los datos obtenidos arrojan que el 58.85\% de los encuestados tiene expectativas favorables porque se hace necesario estructurar un nuevo modelo de gestión operativa para la unidad de emprendimiento, que imprima identidad a cada acción promovida en favor del fomento de la cultura de emprendimiento y el empresarismo guajiro; el $22.1 \%$ no manifiesta interés alguno y el 19.1\% muestra una reacción neutral.

Tabla 2. La implementación del nuevo modelo de gestión, favorecería la incubación, la consecución de recursos y el proceso para la creación de empresas

\begin{tabular}{cccc}
\hline \multicolumn{4}{c}{ El proceso de incubación y creación de empresa } \\
\hline & Frecuencia & $\mathbf{\%}$ & \% Acumulado \\
En desacuerdo & 75 & 20.4 & 20.4 \\
Ni de acuerdo, & 56 & 15.3 & 35.7 \\
ni en desacuerdo & & & \\
De acuerdo & 105 & 28.6 & 64.3 \\
Totalmente de acuerdo & 131 & 35.7 & 100 \\
\hline Total & $\mathbf{3 6 7}$ & $\mathbf{1 0 0 , 0}$ & \\
\hline
\end{tabular}

Fuente: Autores (2012).

Desde esta perspectiva, el $64.3 \%$ de los encuestados manifiesta estar totalmente de acuerdo en torno a que el alma mater debe contar con un centro que asuma el desarrollo del emprendimiento como una inversión social con visión de largo plazo, para ampliar el número y calidad de las oportunidades de negocio; el $15.3 \%$ se mantiene neutral y el $20.4 \%$ expresa desacuerdo por las experiencias vividas el acompañamiento, consolidación y ejecución de sus planes de negocio. 


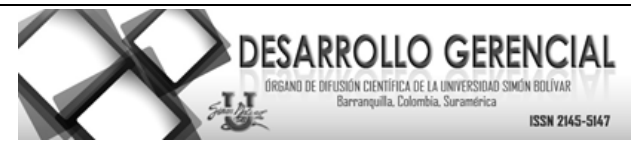

Tabla 3. Apoyo de la Red Regional para el Emprendimiento Guajiro, empresarios, instituciones gubernamentales y financieras proceso de incubación y creación de empresa

\begin{tabular}{lccc}
\hline \multicolumn{3}{c}{ Apoyo de la Red Regional para el Emprendimiento Guajiro } \\
\hline & Frecuencia & $\mathbf{\%}$ & \% Acumulado \\
En desacuerdo & 63 & 17.2 & 17.2 \\
Ni de acuerdo, & 29 & 7.9 & 25.1 \\
ni en desacuerdo & & & 48.8 \\
De acuerdo & 187 & 23.7 & 100.0 \\
Totalmente de acuerdo & $\mathbf{3 6 7}$ & 51.2 & $\mathbf{1 0 0 , 0}$ \\
\hline Total & &
\end{tabular}

Fuente: autores (2012).

El 74.9\% se muestra seguro del apoyo que brinda la Red Regional para el Emprendimiento Guajiro, los empresarios, las instituciones gubernamentales y financieras; el 7.9\% es indiferente ante este apoyo, por no tener una percepción positiva de los entes que fomentan la cultura del emprendimiento en La Guajira, y el $17.2 \%$ está en desacuerdo porque no existe una política institucional sólida y claramente definida que todo el tiempo respalde las iniciativas que en este sentido se generen. Otro de los argumentos identificados revelan una total desarticulación entre la academia, el sector productivo y los entes gubernamentales, que son, al final, los que diseñan y ejecutan la política para la competitividad y productividad de los sectores de la economía departamental.

Tabla 4. Disponibilidad para formar el talento humano, aprender del emprendimiento y desarrollar competencias para crear empresas de innovación tecnológica

\begin{tabular}{lccc}
\hline \multicolumn{4}{c}{ Disponibilidad para formar el talento humano en emprendimiento } \\
\hline & Frecuencia & $\mathbf{\%}$ & \% Acumulado \\
En desacuerdo & 48 & 13,1 & 13,1 \\
Ni de acuerdo, & 35 & 9,5 & 22,6 \\
ni en desacuerdo & 107 & 29,2 & 51,8 \\
De acuerdo & 177 & 48,2 & 100,0 \\
Totalmente de acuerdo & $\mathbf{3 6 7}$ & $\mathbf{1 0 0 , 0}$ & \\
\hline Total & & &
\end{tabular}

Fuente: autores (2012).

El 77.4\% de los encuestados señala un gran interés en aprender del emprendimiento, puesto que el alma mater cuenta con una comunidad académica claramente comprometida con el ejercicio formativo en la actividad emprendedora, en lo que tiene que ver con el trabajo de aula y la investigación tanto básica como aplicada; el 9.5\% no tiene interés alguno porque solo se aborda la etapa de sensibilización para la construcción del plan de negocio sin nada más. De la misma manera, el 13.1\% expone su desacuerdo porque no existe una política que integre las facultades y los programas académicos con las perspectivas 


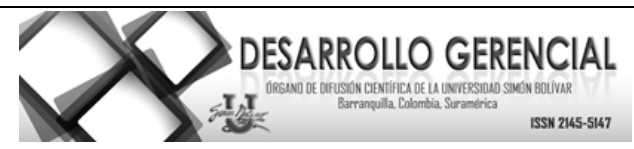

de desarrollo del gobierno (diseñador y ejecutor de la política), los requerimientos del sector productivo y las entidades financieras de apoyo al fomento del emprendimiento.

Tabla 5. El modelo de gestión existente es bueno

\begin{tabular}{lccc}
\hline \multicolumn{4}{c}{ El modelo de gestión existente es bueno } \\
\hline & Frecuencia & $\mathbf{\%}$ & \% Acumulado \\
En desacuerdo & 184 & 50,1 & 50,1 \\
Ni de acuerdo, & 91 & 24,8 & 74,9 \\
ni en desacuerdo & 32 & 8,7 & 83,7 \\
De acuerdo & 60 & 16,3 & 100,0 \\
Totalmente de acuerdo & $\mathbf{3 6 7}$ & $\mathbf{1 0 0 , 0}$ & \\
\hline Total &
\end{tabular}

Fuente: autores (2012).

De acuerdo con la información recopilada, el $50.1 \%$ de los participantes está inconforme con el modelo de gestión existente porque no lo conoce o no ha sido ampliamente difundido en los diferentes estamentos de la comunidad universitaria ni en los medios de comunicación masiva que existen en la localidad; el $24.8 \%$ es indiferente y solo el $25 \%$ se muestra satisfecho con el actual modelo de gestión porque le ha permitido cumplir con el sueño de presentar su idea de negocio para incubación. Además, todos informan que por correo electrónico reciben las diferentes convocatorias que se envían a nivel local, regional, nacional e internacional.

Tabla 6. Análisis correlacional entre las expectativas y percepción del modelo de gestión

\begin{tabular}{llcc}
\hline & \multicolumn{2}{c}{ Tabla de correlaciones } & Expectativas por el \\
& & $\begin{array}{c}\text { Percepción del } \\
\text { nuevo modelo operativo } \\
\text { modelo de gestión } \\
\text { existente }\end{array}$ \\
\hline & $\begin{array}{l}\text { Correlación de Pearson } \\
\text { Sig. (bilateral) }\end{array}$ & 1 & $\mathbf{7 9 6}$ \\
$\begin{array}{l}\text { Expectativas por el nuevo } \\
\text { modelo operativo }\end{array}$ & $\begin{array}{l}\text { Suma de cuadrados y productos } \\
\text { cruzados }\end{array}$ & 489,384 &, 000 \\
& Covarianza & 1,337 & 374,757 \\
& $\mathrm{~N}$ & 367 & 1,024 \\
Percibo del modelo de gestión & Sig. (bilateral) & $\mathbf{7 9 6}$ & 367 \\
existente & Suma de cuadrados y productos &, 000 & 1 \\
& cruzados & 374,757 & 453,210 \\
& Covarianza & 1,024 & 1,238 \\
\hline
\end{tabular}

Fuente: autores (2012).

En términos generales, el análisis bivariado permitió estimar correlación positiva(r: 0.796) estadísticamente significativa (Sig. ,000), entre las expectativas que tiene la comunidad académica de la universidad por el nuevo modelo de gestión operativa y la forma como se percibe el modelo actual de la 


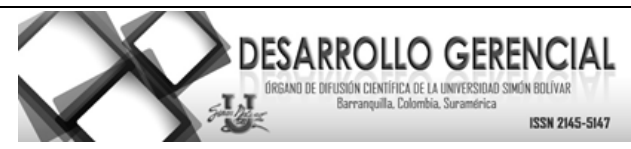

Unidad de Emprendimiento de la Universidad de La Guajira. Al elevar el valor “0.79” al cuadrado, se obtiene el valor " 0.6241 ”, que es el coeficiente de determinación; indica que el $62.41 \%$ de la variación en las expectativas que tiene la comunidad académica de la institución explica la forma como los emprendedores perciben el modelo actual de la Unidad de Emprendimiento.

\section{4.- DISCUSIÓN}

En los últimos reportes de Global Entrepreneurship Monitor GEM, se ha establecido la importancia del emprendimiento en el desarrollo económico, lo cual claramente es una reconocida realidad al punto que se habla de una economía global, en la cual las empresas se orientan hacia el dinamismo, al cambio permanente y a la innovación (Bayona, 2011). Este marco de referencia, constituye un escenario estratégico para la reflexión crítica de los resultados obtenidos.

La mirada de los gobiernos, empresarios y académicos a nivel mundial se ha desplazado hacia las ciudades y a las regiones cuyas intervenciones son decisivas para movilizar la base productiva de una sociedad en una cultura emprendedora, para lo cual es indispensable la interacción de los individuos, empresas e instituciones. En el caso de Colombia, muchas regiones buscan responder a las necesidades de las ciudades de lograr una oferta nacional sólida, que sea capaz de responder a la demanda por servicios de emprendimiento y consolidar un desarrollo empresarial.

Lo anterior gira en torno a un fenómeno que encierra una complejidad particular, dado que ocurre a la vez en tres niveles, y debe operar básica y simultáneamente en las ciudades que han sido emprendedoras como es el caso del nivel básico de los individuos, el nivel entremedio de las empresas y el nivel macro donde se desprenden los factores del entorno. Estos últimos se manifiestan en el individuo a partir del temor al fracaso, percepciones de oportunidades, en donde cada factor en contra conjuga la baja participación de los roles sociales que permiten conocer a individuos emprendedores de éxito (Amit \& Muller, 1994; Chung, 2008).

El término emprendimiento hace referencia a la capacidad que posee un individuo para generar ideas, identificar oportunidades y definir los escenarios adecuados a objeto de convertirlas en realidad a través del proceso de creación de empresas sostenibles; también se relaciona con la capacidad de adaptarse a equipos de trabajo existentes, generar otros nuevos y saber liderar bajo diferentes escenarios teniendo en cuenta sus fortalezas y debilidades (Mises, 1949; Timmons, 1970; Shapero, 1985; Clarysse \& Moray, 2004; González, 2009).

El desarrollo y la estructuración de la Unidad nace inicialmente como la oportunidad para aprovechar una serie de programas y reglamentaciones buscando dinamizar el entorno, en consistencia 


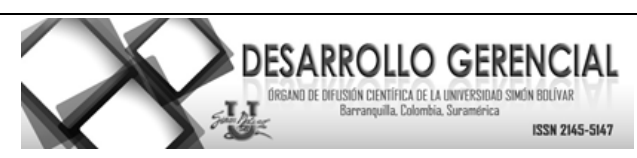

con las reflexiones derivadas del estado del arte en la formación del emprendimiento y los desafíos institucionales en la educación superior planteados por Castillo (1999) y Tobar (1997). Desde esta perspectiva se resalta la necesidad de fortalecer la articulación en la relación de universidad-empresa en el contexto de sociedad-Estado, de forma recíproca y constructiva.

De igual forma las empresas en Colombia tienen una alta participación del emprendimiento motivado por las oportunidades, expectativas de creación de empleos, productos diferenciados y unas tecnologías recientes, cuyo factor en contra está determinado por el sesgo hacia el emprendimiento motivado por las necesidades entre los más jóvenes; pero si se comparan con las instituciones, sus debilidades se manifiestan en los bajos niveles de financiamiento, poca transferencia en tecnología, escaso conocimiento de las implicaciones de la propiedad intelectual en la estrategia empresarial y relativa reducida capacidad de impacto de los programas en iniciativas para desarrollar el emprendimiento en gremios participativos.

Lo anterior ha reflejado la débil participación del entorno empresarial de la Universidad, específicamente de la Unidad de Emprendimiento, puesto que hasta el año 2012 no ha logrado consolidar un plan de negocios desarrollado por los estudiantes de los diferentes programas y así dinamizar la presentación de resultados como proyectos empresariales. Nuevamente se evidencia la necesidad de fortalecer los procesos pedagógicos relacionados con el aprendizaje y fortalecimiento de las competencias asociadas con el emprendimiento empresarial (Andrade \& Bravo, 2009).

En general, de las 367 personas que fueron abordadas, 216 (58.8\%) tienen expectativas por conocer el nuevo modelo de gestión que se propondría para la operatividad de la Unidad de emprendimiento de la Universidad de La Guajira y solo $19.1 \%$ no muestra interés por el mismo. Este resultado es ampliamente compartido con los empresarios y los representantes de los entes gubernamentales, quienes se atreven a manifestar que en La Guajira se requiere despertar el sentido de alerta para percibir las oportunidades de negocio que brinda el medio socioeconómico y la posición geoestratégica que tiene este departamento. Así mismo, se deja claro que la falta de visión de negocio de largo plazo es el motivo por el cual todo intento de crear empresas fracasa en el instante de la gestación del plan de negocio.

El $64.3 \%$, de los encuestados tomaría la iniciativa apoyando la reconversión del modelo de gestión vigentes; además, estaría dispuesto a colocar recursos y talento humano, para apoyar a los emprendedores y promover la gestación de ideas de negocios que fomenten procesos de asociatividad, cooperación, mutualidad, unión y esfuerzo que impulsen la creación de empleos productivos y saquen de la pobreza a muchos de los pobladores del departamento de La Guajira. Estos resultados son importantes desde la óptica de estimular los procesos de asociatividad tal como lo plantean Alburquerque y Valdivieso (2004), Consultores Asociados, CMA (s. f.) y Vegas (2008). 


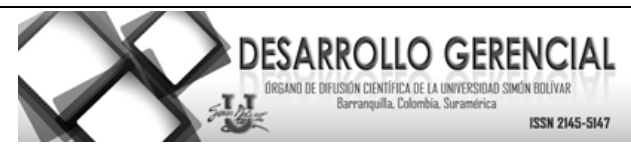

En cuanto si el modelo existente es bueno, la gran mayoría: $74.8 \%$ considera que no lo es o no existe. Hace falta que las acciones que se desarrollan en la unidad en mención sean ampliamente difundidas por televisión, radio, prensa, revista, folleto. Todo lo anterior porque solo se utiliza internet para publicar las convocatorias que realizan otras instituciones de fomento a la cultura del emprendimiento. Estos hallazgos convergen con las recomendaciones de Danhke (1989) y Veciana, Aponte y Urbano (2005) sobre el fortalecimiento de los procesos de comunicación y redes para desarrollar entre jóvenes actitudes hacia el emprendimiento.

Así, a través de su responsabilidad social, y en cumplimiento de los preceptos de fomento introducidos por la Ley 1014/2006, la Unidad de Emprendimiento busca articular sus actividades en la Red de Emprendimiento de La Guajira y otras instituciones, con el objetivo de aunar esfuerzos técnicos, administrativos y financieros para desarrollar actividades conjuntas con el propósito de impulsar el tema en el departamento.

La reconversión del modelo de gestión vigente de la Unidad de Emprendimiento de la Universidad de La Guajira generaría como consecuencia la estructuración del Centro de Desarrollo Empresarial, con miras a fomentar la cultura del emprendimiento desde el quehacer pedagógico de las diferentes facultades y programas académicos de la Universidad de La Guajira. Para tal caso, se ha descrito con anterioridad una serie de variables que permiten elaborar el siguiente diseño de modelo de gestión.

Las universidades del departamento de La Guajira, como centros de educación superior del departamento, deben contar con un Centro de Desarrollo Empresarial que haga acopio de la iniciativas empresariales de los emprendedores y registre aquellos proyectos que son viables técnica, ambiental, legal, social y económicamente en la medida en que lleven a fortalecer la competitividad del departamento y que sean susceptibles de financiación.

Este CDEE estará orientado a la formación de líderes empresariales capaces de crear organizaciones, sean ellas privadas o públicas, con ánimo de lucro o sin él, en cualquier sector de la economía, con cualquier estructura jurídica, con cualquier objetivo, de cualquier tamaño, con cualquier estructura administrativa.

Adicionalmente se deben considerar metodologías probada conforme los niveles de desarrollo de las competencias empresariales (personales y de conocimientos) que se buscan en el proceso; por consiguiente, este trabajo busca formular los elementos generales que en discusión la Universidad puede considerar, y cuya aplicación necesita una transformación enmarcada en un punto de partida del proceso empresarial, a saber:

Desarrollo Gerencial, 7 (1) pp. 140 - 159 Enero-Junio 2015. ISSN: 2145-5147 (On Line). Universidad Simón Bolívar. 


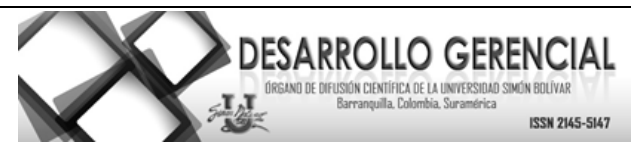

$\checkmark$ La noción de cambio que mueve al ser humano a usar sus capacidades creativas para encontrar nuevas soluciones, o en otros términos: innovar; la noción de acción, que presente eventos, sean ellos materiales y espirituales, y la noción de mejoramiento o de superación, que permita direccionar los cambios hacia la consecución de sus logros y obtención de mejores resultados.

$\checkmark$ Un direccionamiento estratégico claro, bajo el enfoque marcado el emprendimiento y empresarismo municipal.

$\checkmark$ Liderazgo de las instituciones de apoyo al emprendimiento y empresarismo en el departamento, con alto potencial de apoyo para la construcción y puesta en marcha de la Unidad de Emprendimiento.

$\checkmark$ Enfoque hacia ideas de ciencia e innovación tecnológica con alto valor agregado y con viabilidad técnica, económica, ambiental y legal surgidas de los talentos del departamento.

$\checkmark$ Seguimiento de las iniciativas innovadoras por parte de las entidades que guían el proceso de emprendimiento en el ente territorial arriba mencionado.

A partir de los anteriores lineamientos, cabe resaltar que el Centro de Desarrollo Empresarial guarda coherencia con las políticas municipales, departamentales y nacionales en cuanto al emprendimiento se refiere, dado que contribuye a la creación, desarrollo y sostenibilidad de las empresas del departamento de La Guajira. En consecuencia, se espera el crecimiento en la competitividad de los sectores económicos que necesitan el apoyo de talentos que fortalezcan sus procesos a través de proyectos de innovación tecnológica, para lo cual el Centro de Desarrollo Empresarial será una instancia que incentive alianzas entre ellos (Villarán, 2000; Red de Observatorio Regional del Mercado de Trabajo, 2012; Red de Cajas de Herramientas Mypyme, 2007).

Para tal fin, la formación integral del talento humano demanda la construcción de un proyecto de vida empresarial del estudiante para el desarrollo de actitudes emprendedoras. Desde esta perspectiva, se identifican las necesidades de formación y capacitación con el fin de ofrecer a los diferentes programas la asignatura Desarrollo Empresarial como opción para el desarrollo de los seminarios electivos. Indudablemente, el proceso de formación debe ser el elemento que permita el desarrollo de las competencias tanto personales como profesionales para el emprendimiento y la asociatividad (Fernández, 1985; Fontela, Guzmán, Pérez \& Santos, 2006; Herrera \& Giraldo, 2004).

Para esto debe contar con áreas coordinadas por personas expertas en el proceso, que direccionen la formación e incubación y creación de empresas. La Unidad de Emprendimiento Empresarial debe articularse a la Red con el fin de dinamizar el desarrollo del emprendimiento de base tecnológica en el departamento de La Guajira. En la actualidad, la mirada de los gobiernos, empresarios y académicos a 


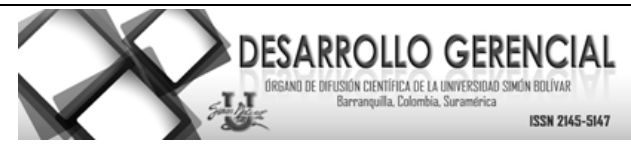

nivel mundial se ha desplazado hacia las ciudades y a las regiones, pues intervenciones son decisivas para movilizar la base productiva de una sociedad a una cultura emprendedora, por lo que resulta indispensable la interacción de los centros educativos, los individuos emprendedores, las empresas o empresarios y las instituciones gubernamentales. En consecuencia, del proceso investigativo se desprenden las siguientes conclusiones:

En cuanto al primer objetivo: Establecer las expectativas del modelo de gestión estratégica para la Unidad de Emprendimiento de la Universidad de la Guajira, se extrae que desde la promulgación de la Ley 1014/2006, el tema del emprendimiento ha sido el centro de interés para la Universidad y el Departamento de La Guajira, y se evidencia que en las políticas de desarrollo económico que reposan en el plan de desarrollo, la agenda de competitividad y la agenda prospectiva del departamento, se incluyen iniciativas para que los gobiernos de turno trabajen por el fomento de la cultura emprendedora.

Sin embargo, desde las expectativas del grupo de involucrados, para que la Unidad de Emprendimiento de la Universidad de la Guajira funcione adecuadamente se requiere de la implementación de una política institucional, seria y concisa, que permita a los procesos de gestión promover estrategias encaminadas a conocer y formar individuos emprendedores de éxitos, generar confianza y credibilidad, conformar capital semilla y estructurar el laboratorio de incubación de empresas. Sus debilidades se manifiestan en la baja participación de los roles sociales, en los bajos niveles de financiamiento, baja transferencia en tecnología, bajo conocimientos de las implicaciones de la propiedad intelectual en la estrategia empresarial y relativamente baja capacidad de impacto de los programas e iniciativas para desarrollar el emprendimiento en gremios participativos.

Todo lo anterior se conjuga con el temor al fracaso y la falta de sentido de alerta que tienen las personas para percibir las oportunidades de negocio que brinda el entorno guajiro. Esta reflexión se ha derivado del análisis de los resultados frente a los planteamientos de la Gobernación de La Guajira (2004), Universidad de La Guajira (2005 y 2010) unidos a los aportes de Gómez (2013), Sobrado y Fernández (2010) y Pereira (2003) sobre la formación y desarrollo del espíritu emprendedor.

En cuanto al segundo objetivo: Identificar los procesos de gestión operativa de la unidad de emprendimiento, se puede concluir que la operatividad de esta área depende de la buena voluntad del rector, quien siempre está presto a brindar el apoyo logístico y económico en los eventos que se organizan para fomentar la cultura del emprendimiento; cabe anotar que los procesos de capacitación encaminados a orientar a los emprendedores que presentan sus proyectos empresariales en el concurso que convoca Fonade a través del Fondo Emprender, aplican la misma metodología que utiliza la Unidad de Emprendimiento y que es desarrollada en diferentes fases operativas, de las cuales la primera es la sensibilización.

Desarrollo Gerencial, 7 (1) pp. 140 - 159 Enero-Junio 2015. ISSN: 2145-5147 (On Line). Universidad Simón Bolívar. 


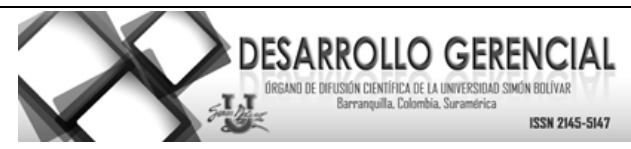

En consecuencia, el mayor esfuerzo se hace con toda la comunidad universitaria y la sociedad en general, en pro de generar un cambio de actitud frente al emprendimiento y al espíritu empresarial. Este proceso se lleva a cabo a través de la organización de diferentes eventos: conferencias, seminarios, ferias empresariales, ruedas de negocios, ruedas financieras, conversatorios de expertos, concursos, video conferencias y foros virtuales entre otras (Clarysse \& Moray, 2004; Del Valle, 2005; Navia \& Herrera, 2007).

Estas recomendaciones convergen con los aportes de autores tales como Schumpeter (1947), Ramos (1998),Stevenson, Gousbeck, Roberts \& Bhide (1999), Angelelli \& Prats (2005), Araya (2006), Sánchez \& Granero (2005), Huertas (2006) y Vesga (2009), quienes resaltan la necesidad de desarrollar diferentes estrategias, para el fomento efectivo de la capacidad emprendedora y los modelos de gestión estratégica en los estudiantes universitarios, a partir de alianzas de este tipo que integren sinérgicamente a la sociedad, el Estado, universidad y empresa.

\section{5.- REFERENCIAS}

Alburquerque, H. \& Valdivieso, L. (2004). Asociatividad empresarial para el fomento del empleo femenino. Un análisis para la región Piura. Tesis de maestría no publicada. Universidad Nacional de Piura, Peru.

Amit, R. \& Muller, E. (1994). Contrasting attributes and attitudes on entrepreneurs and nonentrepreneur. Dallas, TX: Academic Management.

Andrade, M. \& Bravo, W. (2009). Propuesta didáctica para la enseñanza del emprendimiento en el Programa de Administración de Empresas de la Universidad de la Amazonia. Tesis de maestría no publicada, Universidad de la Amazonia, Florencia. Consultado 29 de Junio de 2012 Extraído desde http://www.elitv.org/documentos/tesis/tesis2empresas.pdf.

Angelelli, P. \& Prats, J. (2005). Fomento de la actividad emprendedora en América Latina y el Caribe: Sugerencias para la formulación (Serie de informes técnicos del Departamento de Desarrollo Sostenible; MSM-127). Washington D. C.: Banco Interamericano de Desarrollo. Consultado el 2 de Junio de 2010 Extraído desde http://www.oei.es/etp/fomento_actividad_emprendedora_AL_bid.pdf.

Araya, A. (2006). Cooperación empresarial en Pymes. Tec Empresarial, 1 (1) . 44-48. Consultado 3 de enero 2012 Extraído desde http:// www dialnet.unirioja.es/servlet/fichero_articulo?codigo=2881098.

Bayona, E. (2011). Monitor Global de Emprendimiento: Sección Caribe. Fifth Global Entrepreneurship Monitor Research Conference. Trabajo presentado en el Entrepreneurial Activity: Individual Processes and Contexts, octubre, Cartagena de Indias, Colombia. Consultado 17 de Agosto 2011

Extraído desde http:// www.slideshare.net/redemprendedores/gem-caribe. 
Castillo, A. (1999). Estado del arte en la enseñanza del emprendimiento. First Public Intec- Chile S.A.

Consultado 12 de Enero de 2012 Recuperado desde:

http://www.finam.cl/contenidos/.../estado_arte_emprendimiento.pdf.

Chung, A. (2008). Modelo de cluster empresariales en la gestión de residuos sólidos urbanos. Notas Científicas, 7 (1), 65-69. Consultado 22 de junio de 2012 Extraído desde http://www.sisbib.unmsm.edu.pe/bibvirtualdata/publicaciones/indata/Vol7_n1/pdf/clusters.pdf.

Clarysse, B. \& Moray N. (2004). Un estudio del proceso de formación de equipo empresarial: El caso de la investigación basada en spin-off. Diario del negocio de aventurarse, 19, 55-79.

Congreso Nacional de la República de Colombia. (1990). Ley 29/1990, de 27 de febrero, por la cual se dictan disposiciones para el fomento de la investigación científica y el desarrollo tecnológico y se otorgan facultades extraordinarias. Bogotá: Diario Oficial.

Congreso Nacional de la República de Colombia. (1996). Ley 344/1996, de 27 de diciembre de 1996, por la cual se dictan normas tendientes a la racionalización del gasto público, se conceden unas facultades extraordinarias y se expiden otras disposiciones. Bogotá: Diario Oficial.

Congreso Nacional de la República de Colombia. (1999). Ley 550/1999, de 30 de diciembre. Bogotá: Diario Oficial.

Congreso Nacional de la República de Colombia. (2000). Ley 590/2000, de 10 de julio. Bogotá: Diario Oficial.

Congreso Nacional de la República de Colombia. (2002). Ley 789/20002, de 27 de diciembre. Bogotá: Diario Oficial.

Congreso Nacional de la República de Colombia. (2004). Ley 905/2004, de 2 de agosto. Bogotá: Diario Oficial.

Congreso Nacional de la República de Colombia. (2006). Ley 1095/2006, de 1 de noviembre, por la cual se reglamenta el artículo 30 de la Constitución Política. Bogotá. Diario Oficial. Congreso Nacional de la República de Colombia. (2006). Ley 1014/2006, de 26 de enero, de Fomento a la Cultura del Emprendimiento. Bogotá: Diario Oficial.

Consultores Asociados, CMA. (s. f.). Asociatividad estratégica para el desarrollo. Colombia: Autor. Consultado 3 de Enero de 2012 Extraído desde http:// www.cmaconsultores.com/esp/doc/clusters_SENA_colombia.pdf.

Danhke, G. (1989). Investigación y comunicación. En C. Fernández \& G. Dahnke (Comps.), La comunicación humana: Ciencia Social. México: McGraw Hill.

Del Valle, M. (2005). Forjando emprendedores: Las incubadoras de empresas del Ecuador.

(S. L.): CAF. Consultado 7 de octubre de 2011. Extraído desde http:// www.publicaciones.caf.com/media/1360/180.pdf. 


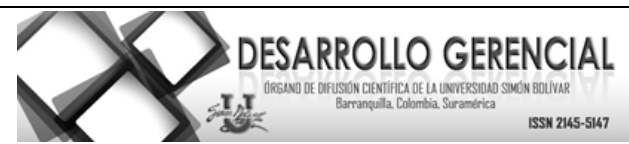

Departamento Nacional de Planeación. (2007). Agenda Interna para la Productividad y la

Competitividad: Documento regional, La Guajira. Bogotá: Autor. Consultado 20 de mayo de 2012

Extraído desde http://www.comisionesregionales.gov.co/descargar.php?id=61366.

Departamento Nacional de Planeación. (2010). Plan Nacional de Desarrollo 2010-2014: Prosperidad

Para Todos. Bogotá: Autor. Consultado 2 de abril de 2012 Extraído desde http://www.dnp.gov.co/LinkClick.aspx?fileticket=4-J9V-FE2pI\%3D\&tabid=1238.

Fernández, C. (1985). Involucramiento en el trabajo. Trabajo presentado en IV Congreso Mexicano de Psicología UNAM, Ciudad de México, México 2011.

Fontela, E.; Guzmán, J.; Pérez, M. \& Santos, F. (2006). El arte de la previsión empresarial. Madrid: Universidad Antonio de Nebrija.

Gámez, J. (2009). Aproximación a los modelos de emprendimiento. Consultado 2 de junio 2011 Extraído desde http:// www. usbbog.edu.co/Nuestra_Universidad/.../M31_art7.pdf.

Garzón, M. (2008). Propuesta de modelo de emprendimiento corporativo o intra emprendimiento para la innovación., pp. 1-22. Consultado 3 de julio de 2012 Extraído desde http:/www.octi.guanajuato.gob.mx/sinnco/formulario/MT/.../MT5_GARZON.pdf.

Gobernación de La Guajira. (2004). Vamos todos a reiniciar La Guajira: Plan de Desarrollo 2.004-2.007. Consultado 10 de noviembre de 2011 Extraído desde http://www.lajaguadelpilarlaguajira.gov.co/apc-aa-files/33323235313439313266656364383934/pd_la_guajira.pdf.

Gómez, A. (2013). Competitividad y cluster, de la teoría a la práctica. Industria forestal. Consultado 5 de mayo de 2012 Extraído desde http://www.gremialforestal.com/articulos/competitividad_y_cluster_de_la_teoria_a_la_practica.pdf.

González, G.; Ruiz, O.; Orozco, D.; Zuleta, Z. \& Morales, S. (2004). Unidad emprendimiento empresarial de la Universidad de Antioquia: Modelo de apoyo a la creación de empresas en una universidad pública latinoamericana. Iberia: Universidad de Valencia. Consultado 10 de diciembre de 2011

Extraído desde http://www.uv.es/motiva/libromotiva/29GonzalezMorales.pdf.

González, L. (2009). Asociatividad alternativa para el desarrollo sostenible en el sector agrícola. Corporación de Organizaciones Productoras de Cacao Nacional Fino de Aroma del Ecuador. Consultado 3 de enero 2012 Extraído desde www.roundtablecocoa.org/.../CONCACAO\%20$\% 20$ Laura\%20Gonz.

Hernández, R., Fernández, C. \& Baptista, P. (2003). Metodología de la investigación: Diseños no experimentales. México: McGraw-Hill.

Herrera, A. \& Giraldo, O. (2004).Uso de un modelo asociativo para el desarrollo del cluster floricultor Colombiano. Bogotá: Universidad de los Andes. Consultado 4 de mayo de 2012 Extraído desde http://www revistaing.uniandes.edu.co/pdf/Rev19-11.pdf.

Desarrollo Gerencial, 7 (1) pp. 140 - 159 Enero-Junio 2015. ISSN: 2145-5147 (On Line). Universidad Simón Bolívar. 
Huertas, E. (1996). Desarrollo institucional en educación para la prevención y atención de desastres. Tesis de maestría no publicada, Universidad Nacional, Medellín.

Institución Universitaria-ITM. (2010). Diseño de la planeación y planificación de unidades de emprendimiento (Informe de avance, Ciudad E05). Consultado 5 de marzo de 2012 Extraído desde http://www.culturaemedellin.gov.co/sites/CulturaE/ciudade/comunidadacademica/Biblioteca\%20de\% 20Documentos/PlaneacionEstrategica.pdf.

Kerlinger, F. \& Lee, H. (2002). Investigación del comportamiento: Métodos de investigación en ciencias sociales. México: McGraw-Hill.

Krishnaswami, S. \& Subramaniam, V. (1999). Asimetría de la información, la valoración y la toma de decisiones corporativas spin-off. Journal of Financial Economics, 53, 73-112.

Lanzas, A., Lanzas, V. \& Castaño, J. (2006). Modelo administrativo para una unidad de emprendimiento en instituciones públicas de educación superior: Caso Universidad Tecnológica de Pereira. Scientia Et Technica, XII (30), 239-249. Consultado \& de abril de 2012 Extraído desde http://redalyc.uaemex.mx/redalyc/src/inicio/ArtPdfRed.jsp?iCve=84920491042.

Lanzas, V., Lanzas, F. \& Lanzas, A. (2009). Propuesta para medir el perfil de los emprendedores de base tecnológica. Scientia Et Técnica, XV (43), 267-272. Consultado 4 de abril de 2012 Extraído desde http://redalyc.uaemex.mx/src/inicio/ArtPdfRed.jsp?iCve=84917310047.

Ledesma, R., Molina, G. \& Valero M. (2002). Análisis de consistencia Interna mediante Alfa de CROBACH. Un programa basado en graficas dinámicas. Psico-USF, 7 (2), 143-152.

Luna, J., Requena, F., Femia, P., Martin, A. \& Miranda, M. (2007). Introducción al manejo del programa SPSS 12.0. Consultado 5 de abril de 2012.. Extraído desde http://www.ugr.es/ bioest/manual_spss.pdf.

Mendoza, J. (2006). Innovación por lo alto: Imaginación y acción en la empresa. Bogotá: Asesores del 2000.

Mises, L. (1949). La acción humana. New Haven: Yale University Press.

Montañés, C. \& Tovar, J. (2009). en el XX Congreso Latinoamericano Sobre Espíritu Empresarial, Universidad ICESI Bogotá. 2011 Características de las unidades universitarias para el apoyo a la creación de empresas y propuesta de modelo para la Universidad Distrital Francisco José de Caldas. Trabajo presentado. Consultado 6 abril de 2012 Extraído desde http:// www.icesi.edu.co/clee2009/.../oct8/.../ppt_p2_carlos_montañez_ii.pd...

Montoya, E. (2009). La creación de spin-offs de base académica-investigativa por iniciativa de los estudiantes: El caso de la Universidad Nacional de Colombia sede Medellín. Tesis de maestría no publicada, Universidad Nacional, Bogotá, Colombia. 
Navia, G. \& Herrera, A. (2007). Construcción de un modelo de gestión social para empresas comunitarias, caso: Mercados móviles de la ciudad. Entramado, 3 (1), 14-23.

Pereira, F. (2003). Reflexiones sobre algunas características del espíritu emprendedor colombiano. Economía , Gestión Y Desarrollo, 1, 9-26.

Ramos, J. (1998). Una estrategia de desarrollo a partir de los complejos productivos (clusters) en torno a los recursos naturales. Santiago de Chile: Cepal.

Rasmussen, E. (2006). Spin-off venture creación en un contexto universitario: una visión de proceso

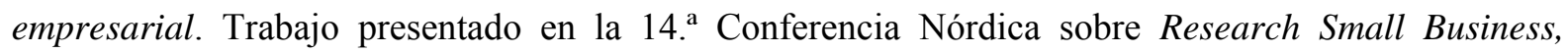
Estocolmo, Suecia. Consultado 8 abril de 2012 Extraído desde http://www.ncsb2006.se/download_title.htm.

Red de Observatorio Regional del Mercado de Trabajo. (2012). Diagnóstico del mercado laboral de La Guajira. Riohacha: Editorial Universitaria, Universidad de La Guajira.

Red de Cajas de Herramientas Mi pyme. (2007). Como iniciar mi empresa. Consultado 10 de mayo 2012 Extraído desde http://www.infomipyme.com/Main.ASP?T=P\&P=2179.

Rodeiro, D.; Fernández, S.; Otero, L. \& Rodríguez, A. (2008). La creación de empresas en el ámbito universitario: Una aplicación de la teoría de los recursos. Cuadernos de gestión, 8(2), 11-28.

Ruiz, O. (2009). El camino hacia los Spin Offs en Medellín Colombia: Experiencias vividas desde la Universidad de Antioquia. Consultado 11 de mayo 2012 Extraído desde http://www.uv.es/motiva2/Ponencias\%20Motiva2009/docs/107.pdf.

Sampieri, R., Collado, C. \& Baptista, P. (2003). Metodología de la Investigación. México: Mc Graw-Hill. Sánchez, M. \& Granero, J. (2005). Calidad total: Modelo EFQM de excelencia. Madrid: Fundación Confemetal.

Schumpeter, J.A. (1947). Capitalismo, socialismo, y democracia (3. ${ }^{a}$ ed.). New York: Harper y Brothers.

Shane, S. (2004). Académico empresarial-University spin-offs y la creación de riqueza. Massachusetts: Edward Elgar Publishing, Inc.

Shapero A. (1985). ¿Por qué el espíritu empresarial? Una perspectiva mundial. Diario de los pequeños. Bussiness Managemet, 1 (1) 1-5.

Sobrado, L. \& Fernández, E. (2010). Competencia emprendedora y desarrollo del espíritu empresarial en los centros educativos. Educación XXI, 13 (1), 15-38.

Stevenson, H.; Gousbeck, H.; Roberts, M. \& Bhide, A. (1999). New business ventures and the entrepreneur. Boston: Irwing McGraw-Hill.

Timmons, J. A. (1970). Nueva empresa: espíritu empresarial. (S. L.): Irwin Homeworod.

Tobar, F. (1997). Desafíos en las organizaciones institucionales, Anlis: Proyecto de Fortalecimiento de la Gestión. Buenos Aires: Mímeo. 
Torres, A. (2008). Simulación discreta: Introducción a la Simulación. Manizales: Universidad de Manizales.

Universidad de Amazonia. (2011). Unidad de Emprendimiento. Extraído el 1 de mayo de 2011 desde http://www.uniamazonia.edu.co/v9/index.php/es/facultades/facultad-de-ciencias-contableseconomicas-y-.

Universidad de Antioquia. (2012). Memoria del Encuentro de Vicerrectores de Investigación de Universidades Públicas Colombianas: ¿Pueden las Universidades Públicas Crear Spin-Off? Medellín: Autor. Extraído el 9 de febrero de 2012 desde http://www.udea.edu.co/portal/page/portal/BibliotecaInvestigacion/InformacionGeneral/utilidades/doc umentos/Relatoria\%20Spin\%20Off.pdf

Universidad de La Guajira. (2003). Proyecto Educativo Institucional, Universidad de La Guajira, Acuerdo N. ${ }^{\circ}$ 009/2003. Consultado 18 de noviembre del 2011 Extraído desde http://www.uniguajira.edu.co/unigua/hermesoft/portal/...1/.../arc_1757.pdf.

Universidad de La Guajira. (2005). Agenda Prospectiva de la Universidad de la Guajira 2004-2014. $\begin{array}{lllllll}\text { Extraído el } & 18 & \text { de } & \text { enero } & \text { de } & 2012 & \text { desde }\end{array}$ www.uniguajira.edu.co/unigua/hermesoft/portal/...1/.../arc_2281.pdf.

Universidad de La Guajira. (2010). Plan de Desarrollo Universidad de La Guajira, 2010-2013: Academia y Proyección Social. Riohacha: Autor. Consultado 18 de noviembre 2011 Extraído desde http:// www.uniguajira.edu.co/unigua/hermesoft/portal/...1/.../arc_2784.pdf

Universidad de los Andes, Icesi, Universidad del Norte \& Universidad Javeriana. (2010). Reporte GEM Colombia 2010.Consultado 19 de noviembre 2011 Extraído desde http://www.gemconsortium.org/docs/download/2207.

Universidad de los Andes, Icesi, Universidad del Norte \& Universidad Javeriana. (2006). Reporte GEM Colombia 2006. Consultado 19 de noviembre 2011Extraído desde http:// www.icesi.edu.co/.../contenido/.../GEM\%20COLOMBIA\%2020061.p...

Universidad de San Buenaventura. (2007). Proyecto Educativo Bonaventuariano PEB. Bogotá D.C.: Consejo de Gobierno. Consultado 20 de noviembre 2011 Extraído desde http://beta.usbcali.edu.co/sitefiles/files/PEB.pdf.

Veciana, J., Aponte, M. \& Urbano, D. (2005). University students' attitudes towards entrepreneurship: A two countries comparison. International Entrepreneurship and Management Journal, 1 (2), 165-182.

Vegas, J. (2008). Asociatividad: Proyecto de Cooperación UE-Perú / PENX. Consorcio Asecal Mercurio Consultores. Consultado 20 de noviembre 2011 Extraído desde http://www.mincetur.gob.pe/comercio/ueperu/consultora/docs_taller/Presentaciones_Tumbes_y_Piura 1.2.1.2.F1\%20Asociatividad\%2020080912.pdf. 


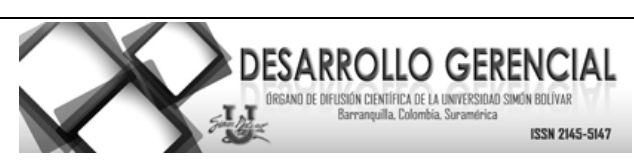

Vesga, R. (2009). Emprendimiento e innovación en Colombia: ¿Qué nos está haciendo falta? Bogotá: Universidad de los Andes. Consultado 19 de noviembre 2011 Extraído desde http://www.cenired.org.co/files/memorias2/3/rafael.pdf.

Villarán, F. (2000). Las pymes en la estructura empresarial peruana. Lima: SASE.

Yin, R. (1989). Investigación estudio de caso: Diseño y métodos. Investigación social aplicada. Métodos, 5. Londres: Sage Publications. 